\title{
THE CONVOLUTIONAL APPROACH TO THE ESTIMATION OF SPECTRAL MAXIMA DIRECTLY FROM THE AUTOCOVARIANCE FUNCTION
}

\author{
HELEN HUTCHENS and R. G. KEATS
}

(Received 11th June 1969)

Communicated by $P$. D. Finch

\section{Introduction}

It is common practice for research workers in a great number of widely differing fields, to gather vast amounts of experimental data (see [1 -3], for example). These data are then analysed, using various statistical or other techniques, in an attempt to obtain information concerning the phenomenon being studied. One important source of such information is evidence of oscillations in the data collected. Various techniques for revealing frequencies of oscillations are available (see [4], for example); they usually involve some form of data processing. In many cases the collected data are records of one or more realisations of a stationary stochastic process, and the frequencies of interest appear as local maxima or peaks in the spectrum of the process [5]. In this paper a method for determining the position of the maximum spectral value directly from the autocovariance function is presented and discussed.

One widely used method for estimating the spectral density function from experimental data is that described by Blackman and Tukey [4]; an essential step in their method being the determination of an apparent autocovariance function. However in some situations the main interest in the spectral density function is the position of its maximum value [6]. In such cases it is not necessary to estimate the spectral density function and the technique described in this paper may then be useful.

\section{The convolutional approach}

Let $\rho(\tau)$ be the-autocovariance function of a real continuous parameter stochastic process which is wide sense stationary and has zero mean. Let $\rho(\tau)$ be continuous and absolutely integrable so that the corresponding spectrum $S(\omega)$ exists and

$$
S(\omega)=\int_{-\infty}^{\infty} \rho(\tau) e^{i \omega \tau} d \tau
$$


Only those processes whose spectra are rational will be considered i.e. spectra which can be expressed as the ratio of two polynomials with real coefficients, the degree of the denominator being at least two higher than the degree of the numerator. The spectra are also assumed to have no zeros on the real axis. Local maxima of such spectra will be called peaks and the so-called periods will be denoted by $T_{i}$, so that $T_{i}=2 \pi / \omega_{i}, \omega_{i} \neq 0$, where $\omega_{i}$ are the peaks in the spectrum.

Since

$$
S(\omega)=\int_{-\infty}^{\infty} \rho(\tau) e^{i \omega \tau} d \tau
$$

then

$$
S^{2}(\omega)=\int_{-\infty}^{\infty} \rho(\tau) * \rho(\tau) e^{i \omega \tau} d \tau
$$

where

$$
\begin{aligned}
\rho(\tau) * \rho(\tau) & =\int_{-\infty}^{\infty} \rho(\tau-u) \rho(u) d u \\
& =\frac{1}{2 \pi} \int_{-\infty}^{\infty} S^{2}(\omega) e^{-i \omega \tau} d \omega .
\end{aligned}
$$

Define $C_{2^{n}}(\cdot)$ such that

$$
\begin{aligned}
& C_{2^{\mathrm{o}}}(\tau)=\rho(\tau), \\
& C_{2^{n}}(\tau)=C_{2^{n-1}}(\tau) * C_{2^{n-1}}(\tau), \quad n \geqq 1 .
\end{aligned}
$$

Then in general

$$
S^{2^{n}}(\omega)=\int_{-\infty}^{\infty} C_{2^{n}}(\tau) e^{i \omega \tau} d \tau
$$

where

$$
C_{2 n}(\tau)=\frac{1}{2 \pi} \int_{-\infty}^{\infty} S^{2^{n}}(\omega) e^{-i \omega \tau} d \omega
$$

In addition, since the peak frequencies of $S^{2^{n}}(\omega)$ are solutions of $d / d \omega\left(S^{2^{n}}(\omega)\right)=$ $2^{n} S^{2^{n}-1}(\omega) S^{\prime}(\omega)=0$, they are the same as the peak frequencies of $S(\omega)$, since $S(\omega)$ and hence $S^{2^{n}-1}(\omega)>0$, all $\omega$.

Suppose $S(\omega)$ has a dominant peak, i.e. a peak with the largest magnitude, .t $\omega_{0}>0$. Define $T_{\omega_{0}}$ as the period in the underlying process corresponding to $\omega_{0}$ It will be shown that

$$
\lim _{n \rightarrow \infty} 4 T_{1 .}=T_{\omega_{0}}
$$

where $T_{n}$ is the smallest positive value of $\tau$ for which $C_{2^{n}}(\tau)=0$. However when $\omega_{0}=0$, i.e. when $S(\omega)$ has its maximum at the origin, $C_{2 n}(\tau)$, for 'sufficiently large' $n$, remains positive for arbitrarily large $\tau$.

The following three theorems provide the core of the convolutional approach. 
THEOREM 1. If $S(\omega)$ is the rational spectrum of a real stationary stochastic process with zero mean, and $S\left(\left|\omega_{0}\right|\right)>S(\omega)$, all $|\omega| \neq\left|\omega_{0}\right|, \omega_{0} \neq 0$, then for every $\varepsilon, 0<\varepsilon<\frac{1}{2}\left|\omega_{0}\right|$, there exists an $N$ such that for $n>N$

(i) $C_{2^{n}}(\tau)=\frac{1}{\pi} \int_{0}^{\infty} S^{2^{n}}(\omega) \cos \omega \tau d \omega>0, \quad 0 \leqq \tau \leqq \frac{\pi}{2\left(\left|\omega_{0}\right|+\varepsilon\right)}$,

and

(ii) $C_{2^{n}}(\tau)=\frac{1}{\pi} \int_{0}^{\infty} S^{2^{n}}(\omega) \cos \omega \tau d \omega=0$

for at least one $\tau$ in the interval

$$
\frac{\pi}{2\left(\left|\omega_{0}\right|+\varepsilon\right)}<\tau<\frac{\pi}{2\left(\left|\omega_{0}\right|-\varepsilon\right)} .
$$

THEOREM 2. If $S(\omega)$ is the rational spectrum of a real stationary stochastic process with zero mean, and $S(0)>S(\omega)$, all $\omega \neq 0$, then for every $T>0$ there exists an $N$ such that for $n>N$,

$$
C_{2^{n}}(\tau)=\frac{1}{\pi} \int_{0}^{\infty} S^{2^{n}}(\omega) \cos \omega \tau d \omega>0, \quad 0 \leqq \tau<T .
$$

THEOREM 3. Under the conditions of theorem 1.

$$
\operatorname{Lim}_{n \rightarrow \infty} \frac{C_{2^{n}}(\tau)}{C_{2^{n}}(0)}=\cos \omega_{0} \tau
$$

A proof of theorem 1 is given by Hutchens [7] while the other two theorems may be proved in a similar way. However they become obvious once it is realised that $S^{2^{n}}(\omega) / C_{2^{n}}(0)$ behaves, for large $n$, like $\pi\left[\delta\left(\omega-\omega_{0}\right)+\delta\left(\omega+\omega_{0}\right)\right]$ where $\delta(\cdot)$ is the Dirac delta function.

Thus, knowing $\rho(\tau)$, the dominant peak frequency of $S(\omega), \omega \geqq 0$, can be determined as follows. If $T_{n}$, the smallest positive value of $\tau$ for which $C_{2^{n}}(\tau)=0$, does not tend to a finite limit as $n \rightarrow \infty, S(\omega)$ has its maximum value at the origin. On the other hand, if $T_{n}$ converges then, for $n$ 'sufficiently large', $T_{n}$ approximates $\pi / 2 \omega_{0}$, where $\omega_{0}$ is the dominant peak frequency of $S(\omega), \omega>0$. Similarly $T_{\omega_{0}}$, the period in the underlying process corresponding to $\omega_{0}$, can be estimated as $4 T_{n}$ for $n$ 'sufficiently large'.

\section{Two theoretical examples}

Two theoretical examples of the application of the convolutional approach will be discussed in this section; a practical application is given in the next section. The first example has a spectral peak at the origin and the second at $\omega=1.19$, while the statistical properties of the two processes considered are: 
(i) $\rho(\tau)=e^{-2|\tau|} \cos \tau$,

$$
S(\omega)=\frac{4\left(\omega^{2}+5\right)}{\left(5-\omega^{2}\right)^{2}+4 \omega^{2}} ;
$$

(ii) $\rho(\tau)=e^{-3|\tau|} \cos 2 \tau$,

$$
S(\omega)=\frac{6\left(\omega^{2}+13\right)}{\left(13-\omega^{2}\right)^{2}+36 \omega^{2}} .
$$

The dominant peak frequencies given above indicate that $T_{n}$ should increase with out limit for case (i) and should converge to 1.315 for case (ii).

The repeated convolutions were carried out using the CDC 6400 at the University of Adelaide. For this purpose the autocovariance functions were digitised at intervals of 0.05 units over the interval $|\tau| \leqq 15$ and were taken as zero for $|\tau|>15$. It was necessary to incorporate in the programme an instruction to stop when $n$ is 'large enough'. Theorem 3 suggests that these limits be imposed so that $C_{2^{n}}(\tau) / C_{2^{n}}(0)$ approximates a cosine curve. Accordingly the following two criteria for stopping the programme were used:
(i) $\left[\frac{\left[\frac{C_{2^{n}}^{\prime}(\tau)}{C_{2^{n}}(0)}\right]_{\tau=T_{n}}+\frac{\pi}{2 T_{n}}}{\pi / 2 T_{n}}\right]<0.1$;
(ii) $\left[\frac{C_{2^{n}}(\tau)}{C_{2^{n}}(0)}\right]_{\tau=m}<-0.9$,

where $m$ is the smallest positive value of $\tau$ such that $C_{2^{n}}(\tau) / C_{2^{n}}(0)$ is a minimum.

\subsection{Results and conclusions}

Table 1 gives the values of $T_{n}$ for some $n$ for the first example, while table 2 gives the interesting information obtained for the second example.

TABle 1

\begin{tabular}{ll}
\hline$n$ & $T_{n}$ \\
\hline 0 & 1.570 \\
1 & 1.854 \\
2 & 2.280 \\
3 & 2.890 \\
4 & 3.760 \\
5 & 4.998 \\
6 & 6.890 \\
\hline
\end{tabular}


TABLE 2

\begin{tabular}{rlll}
\hline$n$ & $T_{n}$ & {$\left[\frac{C_{2^{n}(\tau)}^{\prime}}{C_{2^{n}}(0)}\right]_{\tau=T_{n}}$} & {$\left[\frac{C_{2^{n}(\tau)}}{C_{2^{n}}(0)}\right]_{\tau=m}$} \\
\hline 0 & 0.782 & -0.202 & -0.021 \\
1 & 0.936 & -0.410 & -0.051 \\
2 & 1.071 & -0.464 & -0.088 \\
3 & 1.222 & -0.592 & -0.130 \\
4 & 1.380 & -0.634 & -0.178 \\
5 & 1.490 & -0.680 & -0.245 \\
6 & 1.540 & -0.754 & -0.347 \\
7 & 1.533 & -0.862 & -0.507 \\
8 & 1.425 & -1.010 & -0.712 \\
9 & 1.360 & -1.100 & -0.870 \\
10 & 1.330 & -1.150 & -0.940 \\
11 & 1.330 & -1.168 & -0.963 \\
12 & 1.330 & -1.164 & -0.961 \\
13 & 1.344 & -1.158 & -0.956 \\
14 & 1.340 & -1.155 & -0.954 \\
15 & 1.342 & -1.152 & -0.953 \\
16 & 1.345 & -1.150 & -0.953 \\
\hline
\end{tabular}

The following conclusions may be drawn from these tables.

1. Table 1 shows that $T_{n}$ rapidly increases for each $n$ and it can be concluded that $\rho(\tau)=e^{-2|\tau|} \cos \tau$ is the autocovariance (autocorrelation) function of a stochastic process whose spectrum has its dominant peak at the origin.

2. Column 2 of Table 2 indicates that $T_{n}$ is converging, thus showing the presence of a period in the underlying process. If the two conditions stated above are considered to give suitable accuracy, then using columns three and four of Table 2, $n$ is 'sufficiently large' when $n=11$. This gives $T_{11}=1.33$ as an estimate of $\pi / 2 \omega_{0}=1.315$.

Thus in each of the examples considered, the convolutional approach gives a good estimate of the dominant peak frequency.

\section{A practical application of the convolutional approach}

In this section a practical illustration of the convolutional approach is given, using data on water levels in an experiment described by Noye [2]. These data were punched on cards and were subsequently analysed on the CDC 3200 at Monash University, Victoria. Basically the programme is the same as that used in the theoretical examples discussed in the previous section. However an additional subroutine was incorporated to remove the mean, and another to compute the initial 
autocovariance function. Since the step for obtaining the autocovariance function is very similar to convolution, the programme is essentially one of repeated convolutions, even when one begins with raw data.

The conditions which the convoluted autocovariance function was required to satisfy, before it was assumed that sufficient accuracy had been obtained, were the same as those stated in section 3 except that the second condition was relaxed to

$$
\left[\frac{C_{2^{n}}(\tau)}{C_{2^{n}}(0)}\right]_{\tau=m}<-0.85
$$

where $m$ is the smallest positive value of $\tau$ such that $C_{2^{n}}(\tau) / C_{2^{n}}(0)$ is a minimum. The value -0.85 was chosen after observing the first output using the original two conditions in the programme.

Three convolutions of the autocovariance function were necessary to meet the chosen conditions, giving $T_{3}=385.41$ minutes $=6.423$ hours. Thus $T=4 T_{3}=$ 25.69 hours. This is in close agreement with the estimate of 25.0 hours obtained by Noye [2] for the dominant period present.

\section{General discussion}

The convolutional approach described in this paper provides a method whereby the period corresponding to the dominant peak of the spectrum may be estimated.

Errors which are likely to occur in this approach are:

(i) recording errors;

(ii) computational errors;

(iii) errors due to a finite number of convolutions;

(iv) errors due to a finite length of record.

All these errors except (iii) have been discussed at length in various texts (see [4], [5], for example), with relation to similar problems. In the present case the accuracy of the estimate is affected by the number of convolutions of the autocovariance function which are performed. Since the number of convolutions which can be carried out in practice is limited, suitable conditions must be incorporated to stop the computer programme. These conditions must be related to the required accuracy and one such set of conditions was described earlier. The argument used by Hutchens [7] in the proof of theorem 1 may be adapted to determine a relation between accuracy and the number of convolutions needed.

\section{References}

[1] G. E. R. Deacon, 'Analysis of sea waves', National Inst. Oceanography. Collected reprints. Paper 26, 1, (1953).

[2] J. Noye, 'A limnological study of the Coorong', Research paper no. 11, Horace Lamb Centre for Oceanographical Research, South Australia, (1967). 
[3] N. S. Wahedra and B. A. P. Tantry, 'Audio frequency spectrum of a radio atmospheric at large distances', J. Atmos. Terr. Phys., 28 (1966), 1227.

[4] R. B. Blackman and T. W. Tukey, The measurement of power spectra, Dover (1958).

[5] J. B. Laning and R. H. Battin, Random processes in automatic control, McGraw-Hill (1956).

[6] E. J. Hannan, Time series analysis, Wiley (1962).

[7] H. Hutchens, 'Period detection by autocovariance analysis', M. Sc. Thesis, University of Adelaide (1969).

Department of Mathematics

University of Melbourne

and

Department of Mathematics

University of Newcastle 